\title{
Analyzing the Effect of Variable Round Time for Clustering Approach in Wireless Sensor Networks
}

\author{
Vipin Pal, Girdhari Singh, and R. P. Yadav
}

\begin{abstract}
As wireless sensor networks are energy constraint networks so energy efficiency of sensor nodes is the main design issue. Clustering of nodes is an energy efficient approach. It prolongs the lifetime of wireless sensor networks by avoiding long distance communication. Clustering algorithms operate in rounds. Performance of clustering algorithm depends upon the round time. A large round time consumes more energy of cluster heads while a small round time causes frequent re-clustering. So existing clustering algorithms apply a trade off to round time and calculate it from the initial parameters of networks. But it is not appropriate to use initial parameters based round time value throughout the network lifetime because wireless sensor networks are dynamic in nature (nodes can be added to the network or some nodes go out of energy). In this paper a variable round time approach is proposed that calculates round time depending upon the number of active nodes remaining in the field. The proposed approach makes the clustering algorithm adaptive to network dynamics. For simulation the approach is implemented with LEACH in NS-2 and the results show that there is $6 \%$ increase in network lifetime, $7 \%$ increase in $50 \%$ node death time and $5 \%$ improvement over the data units gathered at the base station.
\end{abstract}

Index Terms-Wireless sensor network, clustering, energy efficiency, round time.

\section{INTRODUCTION}

Wireless sensor networks [1] [2] are collection of large number of sensor nodes deployed in a field. Advancement in Micro-Electro-Mechanical-System (MEMS) provides small sized and low cost sensor nodes. Sensor nodes are equipped with memory, data processing and sensing unit, wireless communication unit and a battery. Wireless sensor networks have layered architecture as shown in Fig. 1. The end-user is not connected directly to the network but via a base station. Nodes sense the area and send the data to base station via single or multi hop communication. The end user accesses the data from base station.

Wireless sensor networks are deployed in harsh environment and the sensor nodes are limited in resources e.g. limited battery power, small memory capacity. Due to harsh working environment it is quite difficult to recharge or replace the battery of sensor nodes. Sensor nodes consume energy with their operations in field e.g. sensing and processing of data, sending and receiving of data. Among various operations data communication is the most energy

Manuscript received August 27, 2012; revised October 7, 2012.

Vipin Pal and Girdhari Singh are with the Malaviya National Institute of Technology, jaipur, 302017, India (e-mail:vipinrwr@ yahoo.com, girdharisingh@ rediffmail.com)

R P Yadav is with the Rajasthan Technical University, Kota, 324010, India (e-mail: rp_yadav@yahoo.com). consuming. [3] shows that the energy consumed to transmit a $1 \mathrm{~KB}$ data to $100 \mathrm{~m}$ is the same as the execution of 3 million instructions by a MIPS/W operations. The lifetime of wireless sensor network depends upon the sensor nodes. Hence energy efficiency is the main design issue for researchers from the manufacturing level to network protocol level [4].

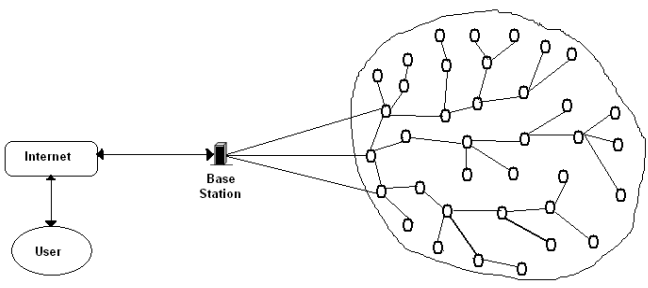

Fig. 1. Wireless sensor network

Clustering [5] [6] is an energy efficient approach in ad-hoc and wireless sensor network. Clustering divides the network in different independent clusters. Each cluster has atleast one cluster head and all nodes in the cluster send data to cluster head. Cluster head applies aggregation technique to reduce the collected data and send the aggregated data to base station. Clustering reduces the communication distance of nodes. Nodes are sending data to nearby located cluster head instead of far away located base station. Cluster head is doing more work than the other nodes in the cluster so consumes energy faster. So the role of cluster head is rotated among all the nodes for network load balance. For energy efficiency duty cycle of sensor nodes should be low. Duty cycle [7] of a node is the ratio of time spends in active mode to total frame time. Clustering prefers TDMA scheduling for node to cluster head communication. Nodes are in active state only if assigned time slot arrives. So clustering lowers the duty cycle of nodes.

After a certain time, known as round time [7], re-clustering is performed. Round time is the total duration of cluster set-up and continuous data processing phase. Energy efficiency of clustering algorithms heavily depends upon the round time. In this paper a variable round time approach is proposed in which the value of round time is made adaptive to network dynamics for energy efficiency. Round time is calculated according to the number of active nodes. Variable round time helps in load balancing of network as the nodes start going out of energy. The work of this paper describes the importance of round time for the energy efficiency of clustering algorithms. The rest of the paper is organized as follows: Section 2 described the clustering schemes in the literature, Section 3 formulates the problem and explains the proposed solution, section 4 provides detail about the network assumptions, parameters for network simulation and 
results and section 5 concludes the work of paper.

\section{LITERATURE REVIEW}

Clustering algorithms avoid long distance communication for energy efficiency of nodes. Clustering algorithms can be classified according to operational mode: Distributed and Centralized. In distributed algorithms, nodes select cluster head and form cluster locally by exchange of information. But in centralized approach, nodes send their status information to a centralized point. The centralized point informs the network about cluster heads and member of clusters of respective cluster heads. Clustering algorithms can be categorized according to state of formed clusters: Dynamic clustering and Static clustering. In dynamic clustering, clusters are formed again and again over the time. But in the static clustering schemes, clusters are formed once throughout the life of network.

LEACH (Low Energy Adaptive Cluster Hierarchy) [8] is fully distributed algorithm. Operation of LEACH is performed in rounds and each round consists of set-up phase and steady phase. In set-up phase cluster heads selection, cluster formation and TDMA scheduling are performed. In steady phase, nodes send data to cluster head and cluster head aggregate the data. Aggregated data is send to base station. After a fix round time, re-clustering is performed. Role of cluster head is rotated to all the sensor nodes to make the network load balance. But the protocol does not guarantee about the number of cluster heads. [9] proposed an improvement over LEACH by selecting the $\mathrm{CH}$ not randomly but considering the remaining energy when the energy level drops below $50 \%$ of the initial energy. Cluster head join process is determined not only by received signal strength but also by the remaining energy of cluster head. The data is sent by a node only if the data satisfies a predefined condition.

LEACH-C [10] is centralized algorithm to form the cluster and to assign duty of cluster head. During the set-up phase, nodes send information about their location and energy level to the base station. The BS formulates clusters using simulated annealing algorithm [11]. The algorithm provides a cluster head to nodes such that nodes minimize their transmission distance and conserve energy. Base station broadcasts message of cluster head ID for each node. The steady phase is same as of LECAH.

The approach in [12] is controlled by base station and a head-set is managed for each cluster to distribute the load of cluster head. Base station determines the suitable number of clusters using that information. Base station broadcasts the information of cluster heads. Then cluster heads construct their clusters and determine the head sets. At one time, only one member of head set is active and receives data from nodes. The task of transmission of aggregated data to base station is distributed uniformly to all the head sets. Along with the data, energy information of nodes is also send to Base station for cluster heads selection of next round.

ADRP in [13] selects a cluster head and set of next heads for upcoming few rounds based on residual energy of each nodes and average energy of cluster. A round of ADRP has two phases: initial phase and cycle phase. In the initial phase, nodes send status of their energy and location to base station.
Base station partitions the network in clusters and selects a cluster head for each cluster along with a set of next heads. In the cycle phase, cluster head aggregates the data and sends to the base station. In the re-cluster stage, nodes transit to cluster head from set of next heads without any assistance from base station. If the set of next heads is empty, initial phase is executed again.

The clustering scheme EAP (Energy-Aware routing Protocol) in [14] provides a new parameter for cluster head selection. A node has higher probability of being a cluster head if it has a higher ratio of residual energy to the average residual energy of all the neighbor nodes in its cluster range. To reduce energy consumption of $\mathrm{CHs}$, a spanning tree is constructed among CHs. The protocol in [15] discusses the problem of unbalanced clusters in the form of number of nodes and total cluster distance. The algorithm partitions the network into clusters such that the solution consumes less communication energy.

\section{Problem Statement AND SOlution}

Clustering is an energy-efficient approach for wireless sensor network. In clustering, round time to re-cluster the network plays a vital role for energy efficiency. Large round time drains more energy from cluster heads because a cluster head has to work for a long time. If the round time is short, nodes will consume energy in re-clustering. Hence the round time should be chosen to take care of above trade-offs.

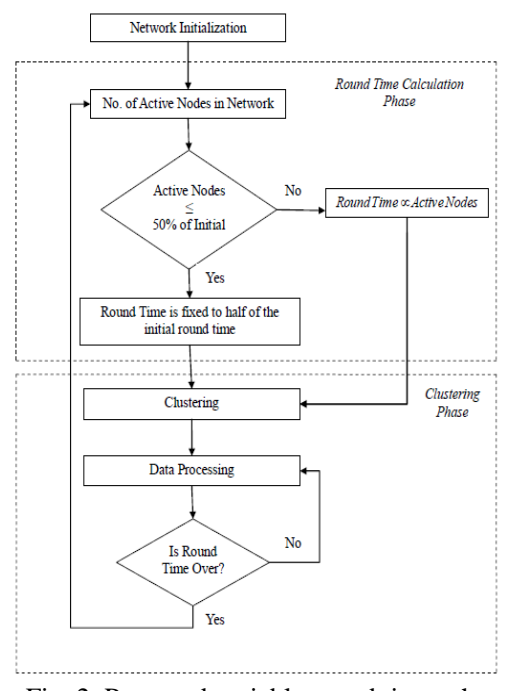

Fig. 2. Proposed variable round time scheme

Most of clustering approaches in the literature have a constant round time for re- clustering of nodes. These approaches calculate round time depending upon the initial number of nodes and fix it for whole network lifetime. But the sensor networks are dynamic in nature. Nodes can be added to the network or some nodes go out of energy. In case of constant round time, if more nodes are added, the round time will not be sufficient to complete the operation and frequent re-clustering will increase the overhead of network. If nodes are not alive, then the round time will be long enough to draw more energy from cluster heads. Hence constant round time makes the network load unbalanced. Hence for a load balanced network round time should be changed dynamically to adjust the network dimensions. 
We have analyzed that in clustering approach at the death of first node $60 \%$ to $65 \%$ of initial energy is consumed and at the time of $50 \%$ node death $80 \%$ of initial energy has been consumed. It shows that nodes are left with very less amount of energy and a selected cluster head may not have enough energy to complete the round. Because initially calculated round might be long enough to drawn all the remaining energy of cluster head which leads to loss of cluster data. So round time should be adaptive to network dynamics.

In our work, round time is calculated dynamically depending upon the number of active nodes in the network. But as the number of active nodes in the network goes down, the round time will be very small causing frequent re-clustering. To avoid frequent re-clustering when the numbers of active nodes are very few then the round time is made to fix such that the round will be completed as well as will avoid frequent re-clustering. Fig. 2 shows the proposed variable round time scheme. The approach makes the clustering algorithm adaptive to network changes.

\section{RESULTS}

The network of different network topologies is simulated in NS-2 [16]. NS-2 is widely used network simulator. It is an event driven network simulator capable of simulate both wired and wireless networks. It has two languages: $\mathrm{C}++$ at the backend while OTcl at frontend. For implementation of LEACH protocol uAMPS [17] project of MIT is patched with NS-2.

\section{A. Energy Model}

The energy used in the simulation is the same used in [8]. For short distance communication free-scale propagation model is used while for long distance communication two-ray ground model is used. Energy required to transmit an 1-bit data over a distance $\mathrm{d}$ is calculated as:

$$
E_{T x}(l, d)= \begin{cases}l E_{\text {elec }}+l_{\varepsilon f r i s s-a m p} d^{2} & d<d_{\text {crossover }} \\ l E_{\text {elec }}+l_{\text {two-ray-amp }} d^{4} & d \geq d_{\text {crossover }}\end{cases}
$$

Energy consumed in receiving the message is calculated as:

$$
E_{R x}(l)=l E_{\text {elec }}
$$

\section{B. Network Assumptions}

Following network assumptions are considered:

1) All sensor nodes are homogenous

2) Nodes are deployed randomly.

3) All nodes are stationary once deployed in the field.

4) Nodes are location aware.

5) There is single base station located outside the field.

6) All nodes have data to send.

7) The nodes were considered to die only when their energy is exhausted. Sudden failure of nodes was not considered.

\section{Network Model}

Different network topologies varying in number of nodes and dimension of area are generated and simulated. Simulation parameters are:

TABLE I: PARAMETER VALUES FOR SiMULATION

\begin{tabular}{ll}
\hline \hline Parameters & Values \\
\hline Network Area & $50 \times 50 \mathrm{~m}^{2}, 100 \times 100 \mathrm{~m}^{2}, 150 \times 150 \mathrm{~m}^{2}$ \\
Number of Nodes & $50,100,200$ \\
Clusters & $5 \%$ \\
Header Packet Size & 25 Bytes \\
Data Packet Size & 500 Bytes \\
Base Station & $75 \mathrm{~m}$ \\
Initial Energy & $2 \mathrm{Joules}$ \\
Bandwidth & $1 \mathrm{Mbps}$ \\
\hline \hline
\end{tabular}

\section{Performance Metrics}

Network Lifetime: Network lifetime can be defined as the time until all nodes are not dead or some predefined conditions are not met. In our work the network will be considered functioning till active nodes are greater than optimal number of cluster heads in network.

50\% Node Death: Time for 50\% node death is an important metric. As summarized in above section at the death of $50 \%$ node most of the energy is consumed. So the protocol should prolong that time for increase in network lifetime.

Data Units Received at Base Station: The sensed data send by nodes to cluster head should reach at base station. The data units received at base station is the measure of quality of network.

\section{E. Performance Analysis}

Three different network topologies are initialized: $50 \times 50 \mathrm{~m}^{2}$ with 50 nodes, $100 \times 100 \mathrm{~m}^{2}$ with 100 nodes and $150 \times 150 \mathrm{~m}^{2}$ with 200 nodes. Fig. 3 shows that there is an improvement in network lifetime for varaible round time LEACH over fixed round time LEACH. There is $7 \%$ improve for $50 \times 50 \mathrm{~m}^{2}$ topolgy, $6.5 \%$ for $100 \times 100 \mathrm{~m}^{2}$ and $6 \%$ for $150 \times 150 \mathrm{~m}^{2}$.

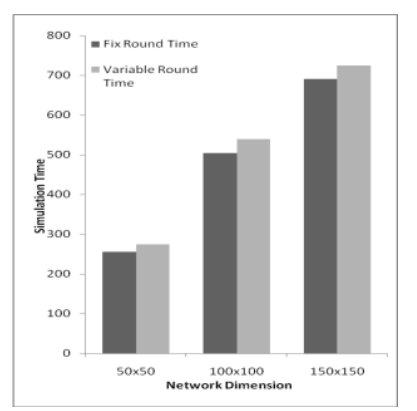

Fig. 3. Comaprison of netwotk lifetime

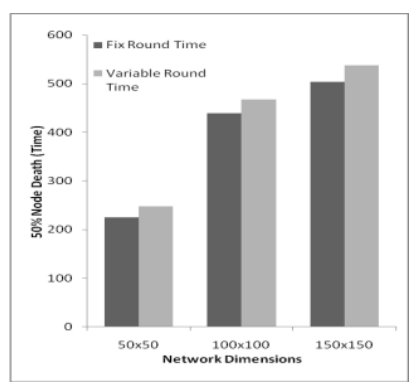

Fig. 4. Comaprison of $50 \%$ node death time 
Fig. 4 shows result for $50 \%$ node death (Time) for the three topologies. There is an improvement of $9 \%$ for $50 \times 50 \mathrm{~m}^{2}, 6 \%$ for $100 \times 100 \mathrm{~m}^{2}$ and $7 \%$ for $150 \times 150 \mathrm{~m}^{2}$.

Fig. 5 shows result of data units received at base station for three topologies. As there is an improvement in network lifetime and $50 \%$ node death time, nodes are having more time to sense the field and send the data to cluster head. There is improvement of $7 \%$ for $50 \times 50 \mathrm{~m}^{2}$ and $5 \%$ for both $100 \times 100 \mathrm{~m}^{2}$ and $150 \times 150 \mathrm{~m}^{2}$.

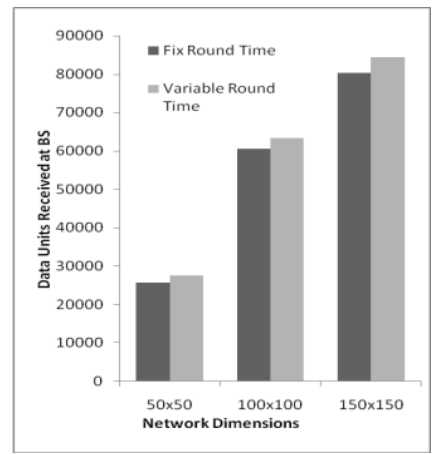

Fig. 5. Comaprison of data units received at BS

Hence the results show that the variable round time scheme according to active number of nodes in network improves the energy efficiency approach of LEACH protocol The above scheme can be implemented with any clustering scheme.

\section{CONCLUSION}

Dynamic nature of wireless sensor network requires adaptability of protocols to the network changes to prolong the network lifetime. In this paper, round time of clustering algorithm is made adaptive to network dynamics by calculating it depending upon the number of alive nodes in the network. Our results show that there is $6 \%$ increase in network lifetime, $7 \%$ increase in $50 \%$ node death time and $5 \%$ improvement over the data units gathered at the base station. In the future, we will also consider the remaining energy of network in round time calculation

\section{REFERENCES}

[1] I. Akyildiz, W. Su, Y. Sankarasubramaniam, and E. Cayirci, "Wireless Sensor Networks: A Survey," Computer Networks, vol. 38, no. 4, pp. 393-422, 2002.

[2] D. Estrin, R. Govindan, J. S. Heidemann, and S. Kumar, "Next Century Challenges: Scalable Coordination in Sensor Networks," in Proc.of MOBICOM, 1999, pp. 263-270.

[3] A. Flammini, P. Ferrari, D. Marioli, E. Sisinni, and A. Taroni, "Wired and Wireless Sensor Networks for Industrial Applications," Microelectronics Journal, vol. 40, pp. 1322-1336, 2009.

[4] G. Anastasi, M. Conti, M. D. Francesco, and A. Passarella, "Energy conservation in wireless sensor networks: A survey," Ad Hoc Network, vol. 7, no. 3, pp. 537-568, 2009.

[5] A. Abbasi and M. Younis, "A survey on Clustering Algorithms for Wireless Sensor Networks," Computer Communication, vol. 30 no. 14-15, pp. 2826-2841, 2007.

[6] D. Wei and H. Chan, "Clustering Ad Hoc Networks: Schemes and Classifications," in Proc. of 3rd Annual IEEE Communications Society on Sensor and Ad Hoc Communications and Networks, 2006, pp. 920-926.

[7] H. Karl, and A. Willig, Protocol and Architecture for Wireless Sensor Networks, Wiley Publication, 2005.

[8] W. R. Heinzelman, A. Chandrakasan, and H. Balakrishnan, "Energy-Efficient Communication Protocol for Wireless Microsensor Networks," in Proc. of the 33rd Hawaii International Conf. on System
Sciences (HICSS '00), IEEE Computer Society, Washington, DC, USA 2000, pp. 8020-8031.

[9] K. Y. Jang, K. T. Kim, and H. Y. Youn, "An Energy Efficient Routing Scheme for Wireless Sensor Networks," in Proc. of International Conf. on Computational Science and its Applications, ICCSA, 2007, pp. 399-404.

[10] W. Heinzelman, A. Chandrakasan, and H. Balakrishnan, "An Application-Specific Protocol Architecture for Wireless Microsensor Networks," IEEE Transactions on Wireless Communications, vol. 1, no. 4, pp. 660-670, 2002.

[11] T. Murata and H. Ishibuchi, "Performance evaluation of genetic algorithms for owshop scheduling problems," in Proc. of the First IEEE Conf. on IEEE World Congress on Computational Intelligence, Evolutionary Computation, 1994, pp. 812-817.

[12] A. S. Zahmati, B. Abolhassani, A. Asghar, B. Shirazi, and A. S. Bakhtiari, An Energy-Efficient Protocol with Static Clustering for Wireless Sensor Networks, 2007.

[13] S. Hussain and A. W. Matin, "Base station assisted hierarchical cluster-based routing," in Proc. of International Conf. on Wireless and Mobile Communications, 2006.

[14] F. Bajaber and I. Awan, "Adaptive decentralized re-clustering protocol for wireless sensor networks," Journal of Computer System Science, vol. 77, no. 2, pp. 282-292, 2011

[15] M. Liu, J. Cao, G. Chen, and X. Wang, "An Energy-Aware Routing Protocol in Wireless Sensor Networks," Sensors, vol. 9, no. 1, pp. 445-462, 2009.

[16] S. Ghiasi, A. Srivastave, X. Yang, and M. Saraafzadeh, "Optimal Energy Aware Clustering in Sensor Networks," Sensors, vol. 2, pp 258-269, 2002

[17] K. Fall and K. Vardhan. The Network Simulator (ns-2). [Online]. Available: http://www.isi.edu/vint/nsnam/

[18] MIT uAMPS project code extensions. [Online]. Available: http://www.mt1.mit.edu/research/icsystems/uamps/research/leach/leac h_code.shtml.

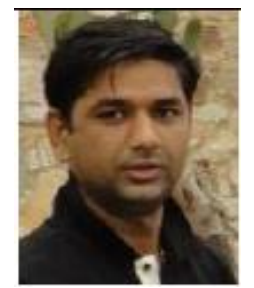

Vipin Pal is a Ph.D scholar at Malaviya National Institute of Technology, Jaipur, India. He completed B.E. in 2005 from Chhotu Ram State College of Engineering, Murthal, India in Computer Science and Engineering and M.Tech. in 2008 from Guru Jambheshwar University of Science and Technology, Hisar, India in Computer Science and Engineering.

His areas of interest are Wireless Sensor Networks, Wireless Ad-Hoc Networks, and Algorithm Design.

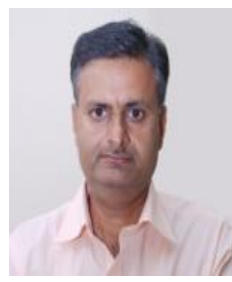

Girdhari Singh is Associate Professor in Computer Engineering Department at Malaviya National Institute of Technology, Jaipur, India. He completes his Ph.D. from Malaviya National Institute of Technology, Jaipur in 2009. He completed B.E. in 1990 from Amaravati University, Amravati, India in Computer Engineering and M.S. in 1996 from BITS Pilani, India in Software Systems. His research interests are Software Engineering and Intelligent Systems

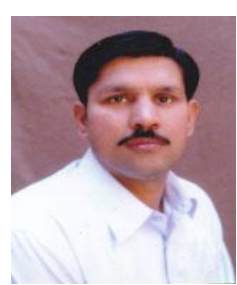

R P Yadav has his initial education in the countryside and later at the Department of Electrical Engineering in Indian Institute of Technology, Delhi, India for his Master of Technology in Integrated Electronics and Circuits in 1987. He earned Ph.D. in Communication Engineering in 2000 from University of Rajasthan, Jaipur, India. He joined Department of Electrical Engineering at the then Malaviya Regional Engineering College, Jaipur (Presently, Malaviya National Institute of Technology) as lecturer in 1987. In Mar. 1993, he was promoted to the post of Reader in Electronics \& Communication Engineering and became Professor in October 2006. Currently he is Vice-Chancellor at Rajasthan Technical University, Kota, India. He is member of various professional bodies including ISTE, BES (India), IEEE and Institution of Electronics \& Telecommunication Engineering (IETE). His research interests are in the areas of Networking, Microwave Communication, MIMO-OFDM and Error Control Coding. 